\title{
Correlación entre las medidas de centralidad de los países y el impacto de sus artículos. Caso de estudio de la investigación sobre biotecnología en Latinoamérica
}

\author{
Guillermo Armando Ronda Pupo * \\ Yesenia Ronda Danta ** \\ Yusleydis Leyva Pupo ***
}

Artículo recibido:

1 de octubre de 2014.

Artículo aceptado:

27 de octubre de 2015.

\section{Resumen}

El objetivo del presente estudio es develar la estructura latente de la red latinoamericana de colaboración regional en la investigación sobre biotecnología y determinar si el impacto de los artículos latinoamericanos sobre biotecnología se relaciona con las medidas de centralidad (grado, intermediación y proximidad) de los países latinoamericanos en la estructura de la red de colaboración regional. Para lograrlo se examinaron 14173 artículos, publicados entre 1988 y 2012, combinando técnicas del análisis de redes sociales con métodos bibliométricos. Los resultados obtenidos muestran que el impacto de los artículos latinoameri-

* Universidad Católica del Norte, Chile. Universidad de Holguín, Cuba. gronda@ucn.cl

** Universidad de Ciencias Médicas, Holguín, Cuba.

$\star \star \star$ Policlínica Alcides Pino Coello, Holguín, Cuba. yusleidysleyva@gmail.com

INVESTIGACIÓN BIBLIOTECOLÓGICA, Vol.30, Núm.69, mayo/agosto, 2016, México, ISSN: 0187-358X. pp. 75-94 
canos sobre biotecnología está correlacionado positivamente con la intermediación de los países, no así con el grado de centralidad o la proximidad. El hallazgo demuestra la importancia de fomentar las redes de colaboración internacional como una vía favorecedora para desarrollar la investigación sobre biotecnología en Latinoamérica.

Palabras clave: Investigación sobre Biotecnología; Grado de Centralidad; Índice Crown; Impacto Científico; Intermediación, Proximidad.

\section{Abstract}

Correlation between a country's centrality measures and the impact of research paper: The case of biotechnology research in Latin America

Guillermo Armando Ronda-Pupo, Yesenia Ronda-Danta and Yusleydis Leyva-Pupo

The aim of this paper is to unveil the latent structure of the Latin American regional biotechnology research collaboration network, as well as to determine whether the impact of a biotechnology paper is correlated to centrality measures (degree, betweenness and closeness) of Latin American countries within the structure of the regional collaboration network. To achieve these objectives, 14,173 Latin American biotechnology papers published between 1988 and 2012 were analyzed, using a combination of social network analysis and bibliometric techniques. Results of the study show the impact of a Latin American biotechnology paper is positively correlated to its country's betweenness, but not to its centrality degree or closeness. These findings show the importance of developing collaboration networks to impel biotechnology research in Latin America.

Keywords: Centrality Degree; g-index; Research on Biotechnology; Scientific Impact. 


\section{INTRODUCCIÓN}

T a producción científica mundial sobre biotecnología se ha incrementado Lde manera significativa en los últimos 30 años. Mientras que en 1990 se publicaron 5427 artículos sobre esta área de investigación en revistas de la base de datos ISI Web of Science, en 2010 esta cifra se cuadriplicó al aparecer 23292 documentos. Resulta impresionante que un solo país, Estados Unidos de América, en 2010 estuvo a 10 artículos de igualar la producción científica mundial de 1990 al publicar 5417 textos sobre biotecnología.

La región de Latinoamérica y el Caribe también experimentó un crecimiento importante en la producción científica sobre biotecnología en los últimos años. En 2010 se incrementó en 12 veces el número de artículos en revistas de la base de datos ISI Web of Science al publicarse 1157 textos contra 93 en 1990. A pesar del vertiginoso crecimiento del número de textos latinoamericanos sobre biotecnología en las revistas de mayor prestigio a nivel mundial en 2010, este incremento sólo representó un $4.96 \%$ de la producción científica mundial en esta área. Este resultado muestra una importante brecha en relación con los países de mayor desarrollo científico en esta categoría de investigación. Por ejemplo, Corea del Sur, ubicada en el lugar 9 del mundo de acuerdo con su producción científica de 2010, sobrepasa en 3 artículos el número de textos publicados por todos los países latinoamericanos juntos en ese año.

El incremento del número de documentos publicados y el creciente interés de los países por desarrollar esta disciplina científica debido al impacto que produce en la sociedad ha atraído el interés para su estudio desde el punto de vista de la cienciometría, lo cual se evidencia en el incremento de las investigaciones sobre el tema (Dalpé, 2002; Glänzel y Zhou, 2010; Huang et al., 2013; McCain, 1995).

Los estudios del impacto de los artículos sobre biotecnología son escasos. Investigaciones previas se han realizado por Dalpé (2002), quien analizó los patrones de citaciones entre artículos y patentes y demostró que no existen diferencias significativas del impacto de las publicaciones que citan a las patentes respecto de los que no las citan. En otro estudio Eslami, Ebadi y Schiffauerova (2013)analizaron los efectos de las redes de colaboración en Canadá en la producción científica y su impacto y encontraron que la estructura de la red de coautoría de los artículos canadienses afecta la producción científica sobre biotecnología. Sin embargo, la estructura de la red no tiene un efecto significativo en el impacto de las patentes generadas por los investigadores. En Latinoamérica se han estudiado las redes de colaboración científica en el campo de la biotecnología en el norte de Brasil (Costa, Da Silva y 
Macedo, 2012). Estos autores demuestran el predominio de la colaboración intrainstitucional y regional sobre la internacional en la investigación sobre biotecnología en el norte de Brasil.

La relación entre las medidas de centralidad y el impacto de los artículos ha sido estudiada en ciencias de la información a nivel de autores (Abbasi, Chung y Hossain, 2012), de instituciones (Abassi, Altmann y Hossain, 2011), investigadores de una misma institución (Cimenler, Reeves y Skvoretz, 2014); en química a nivel de investigadores de un país (Badar, Hite y Badir, 2012, 2014). No se han encontrado antecedentes previos sobre el análisis de la influencia de la centralidad en el impacto de las publicaciones en el área de biotecnología a nivel de países de una región.

En estudios previos Cimenler, Reeves y Skvoretz (2014) analizaron la relación de las medidas de la centralidad con el impacto medido a través del índice de Hirsch (2005). Por su parte, Abbasi, Chung y Hossain (2012; ver también Abbasi, Hossain y Leydesdorff, 2012) midieron dicha relación estudiando el impacto a través del índice $g$ (Egghe, 2006) y Badar, Hite y Badir $(2012,2014)$ miden el impacto mediante la producción científica normalizada. No se encontraron antecedentes sobre el análisis de la relación de las medidas de centralidad con el impacto medido a través del índice Crown, que es precisamente lo que se realiza en el presente estudio.

Así, el resultado de la presente investigación aportará información para el diseño de la estrategia de publicación de los centros de investigación y de los entes financiadores de la investigación científica en la región latinoamericana y el Caribe.

\section{OBJETIVO Y PREGUNTAS DE INVESTIGACIÓN}

El objetivo de la presente investigación es determinar si el grado de centralidad de los países latinoamericanos en la red de colaboración predice el impacto de sus artículos sobre biotecnología. Este objetivo se puede especificar a partir de las siguientes preguntas de investigación:

1. ¿Cuál es la estructura latente de la red de colaboración y la centralidad de los países en la investigación latinoamericana sobre biotecnología?

2. ¿Cuál es el impacto medido a través del índice Crown que alcanzan los artículos científicos latinoamericanos sobre biotecnología por cada uno de los países de la red?

3. ¿Existe relación entre las medidas de centralidad: grado, intermediación y proximidad de los países latinoamericanos en la red de colabo- 
ración académica regional y el impacto de sus artículos sobre biotecnología medido a través del indicador Crown?

Para responder a estas preguntas se han empleado métodos cienciométricos combinados con el análisis de redes sociales. Esta elección posibilita tanto un análisis cuantitativo de la producción científica sobre biotecnología de los países latinoamericanos como un análisis cualitativo, que muestra en una red los países más centrales en la colaboración científica en torno a los artículos sobre biotecnología publicados en revistas de la base de datos ISI Web of Science entre 1988 y 2012.

\section{Marco teóRICO Y ANTECEDENTES}

\section{Relación entre las medidas de centralidad y el impacto de las publicaciones}

Frenken, Hoolzl y De Vor (2005) señalan que las redes de colaboración son una vía efectiva para la producción y la difusión del nuevo conocimiento así como que ambos aspectos contribuyen con el incremento del impacto de las publicaciones.

En la Tabla 1 se presentan los estudios previos que analizan la relación de las medidas de centralidad con el impacto de la investigación. Como se puede apreciar la similitud entre los estudios radica en que todos analizan las tres medidas de centralidad más importantes: grado, intermediación y proximidad. Las diferencias radican en el área estudiada y el indicador seleccionado para medir el impacto. Así Abbasi, Chung y Hossain (2012) y Abbasi, Altmann y Hossain (2011) miden el desempeño mediante el índice g (Egghe, 2006), Cimenler, Reeves y Skvoretz (2014) utilizan el índice de Hirsch (2005) y Badar, Hite y Badir $(2012,2014)$ utilizan el factor de impacto ajustado de la producción científica de investigadores en la disciplina química de Pakistán.

Tabla 1. Estudios previos sobre la relación de centralidad con el desempeño de las publicaciones

\begin{tabular}{|l|l|l|l|}
\hline \multicolumn{1}{|c|}{ Estudio } & Medida(s) de centralidad & Indicador de desempeño & Área de estudio analizada \\
\hline $\begin{array}{l}\text { Abbasi, Altmann y } \\
\text { Hossain (2011) }\end{array}$ & $\begin{array}{l}\text { Grado } \\
\text { Intermediación } \\
\text { Proximidad } \\
\text { Eigenvector }\end{array}$ & Índice g & $\begin{array}{l}\text { 477 artículos sobre } \\
\text { ciencias de la información } \\
\text { de } 5 \text { universidades }\end{array}$ \\
\hline $\begin{array}{l}\text { Abbasi, Chung y } \\
\text { Hossain (2012) }\end{array}$ & $\begin{array}{l}\text { Grado } \\
\text { Ego intermediación }\end{array}$ & $\begin{array}{l}\text { Índice g (Google Acadé- } \\
\text { mico) }\end{array}$ & $\begin{array}{l}10 \text { investigadores de } \\
\text { Ciencias de la información }\end{array}$ \\
\hline
\end{tabular}




\begin{tabular}{|l|l|l|l|}
\hline $\begin{array}{l}\text { Badar, Hite y Badir } \\
\text { (2012) }\end{array}$ & $\begin{array}{l}\text { Grado } \\
\text { Proximidad } \\
\text { Intermediación }\end{array}$ & $\begin{array}{l}\text { Producción científica, } \\
\text { Factor impacto (ISI Web of } \\
\text { Science) }\end{array}$ & $\begin{array}{l}1699 \text { artículos de } 2027 \\
\text { investigadores en el área } \\
\text { de química en Pakistán }\end{array}$ \\
\hline $\begin{array}{l}\text { Cimenler, Reeves y } \\
\text { Skvoretz (2014) }\end{array}$ & $\begin{array}{l}\text { Grado } \\
\text { Intermediación } \\
\text { Proximidad } \\
\text { Eigenvector }\end{array}$ & Índice h & $\begin{array}{l}107 \text { investigadores de la } \\
\text { universidad del Sur de la } \\
\text { Florida }\end{array}$ \\
\hline $\begin{array}{l}\text { Badar, Hite y Badir } \\
(2014)\end{array}$ & $\begin{array}{l}\text { Grado } \\
\text { Proximidad } \\
\text { Intermediación }\end{array}$ & $\begin{array}{l}\text { Producción científica (ISI } \\
\text { Web of Science) }\end{array}$ & $\begin{array}{l}1699 \text { artículos de autores } \\
\text { de Pakistán }\end{array}$ \\
\hline Presente estudio & $\begin{array}{l}\text { Grado } \\
\text { Proximidad } \\
\text { Intermediación }\end{array}$ & Índice Crown & $\begin{array}{l}14173 \text { artículos sobre } \\
\text { biotecnología de 21 países } \\
\text { latinoamericanos }\end{array}$ \\
\hline
\end{tabular}

\section{Relación del grado de centralidad con el impacto}

Abbasi, Altmann y Hossain (2011) reportan la existencia de una correlación positiva entre el grado de centralidad de los autores de 477 artículos sobre ciencias de la información y su impacto (índice $g$ ) $r=, 305, p<0,001$. Posteriormente Abbasi, Chung y Hossain (2012) analizaron la relación de la centralidad de 10 investigadores de ciencias de la información y su desempeño (índice g) y mostraron la existencia de una correlación positiva $r=, 327, p<0,05$ entre grado e impacto.

Asimismo, Badar, Hite y Badir (2012) analizaron la relación entre la centralidad y el desempeño de 2027 investigadores pakistaníes en el área de química y demostraron que la centralidad determina el desempeño de los investigadores en un $56 \% R^{2}=, 568, p<0,01$. Posteriormente Badar, Hite y Badir (2014) complementaron el estudio previo y reportaron que el grado de centralidad de 2027 investigadores en el área de química en Pakistán influye en su desempeño $(\beta=1.056, p<0,01)$.

Por otra parte Cimenler, Reeves y Skvoretz (2014) estudiaron la relación del grado de centralidad con el desempeño medido a través del índice de Hirsh de 107 investigadores de la Universidad de Florida del Sur y concluyeron que existe una influencia positiva del grado de centralidad en el desempeño de dichos investigadores y reportan una relación positiva del grado de centralidad con el desempeño de los investigadores $r=, 422, p>0,01$.

De acuerdo con los resultados previos el grado de centralidad se asocia positivamente con el desempeño de investigadores e instituciones. En la presente investigación se espera que entre mayor sea el número de conexiones que posee un país con otros a través de sus investigadores mayor será su impacto en la investigación científica en el área de biotecnología. El número de relaciones aumenta la capacidad de absorción del país mediante el fomento 
de su capital intelectual, lo cual se daría por el aumento de las capacidades de asumir nuevos conocimientos, técnicas, tecnologías de avanzada, recursos escasos y aprender de la experiencia de los investigadores con mayor desarrollo científico en el área de la biotecnología. Así, como primera hipótesis se propone que existe una correlación positiva entre el grado de centralidad del país latinoamericano en la estructura de la red de colaboración regional y el impacto de sus artículos sobre biotecnología.

\section{Relación de la intermediación con el impacto}

Abbasi, Altmann y Hossain (2011) reportan la existencia de una correlación positiva entre la intermediación de los autores en la estructura de la red de colaboración y su impacto (índice $g$ ) $r=529, p<0,001$. En posterior estudio Abbasi, Chung y Hossain (2012) demostraron la existencia de una correlación positiva $r=, 771, p<0,05$ entre la intermediación de 10 investigadores de ciencias de la información y su impacto.

En el estudio de Badar, Hite y Badir (2012) se reporta que la intermediación de 2027 investigadores pakistaníes en el área de química predice su desempeño de $R^{2}=139, p<0,01$. Sin embargo, Badar, Hite y Badir (2014) reportaron la no existencia de influencia de la proximidad la red de coautoría de 2027 investigadores en el área de química en Pakistán no influye en su desempeño $(\beta=0,014, p>0,01)$. Mientras que Cimenler, Reeves y Skvoretz (2014) encontraron una influencia de la intermediación de los autores en la red de colaboración de 107 investigadores de la Universidad de Florida del Sur en su impacto (índice $b$ ), con la característica que sólo es significativa para los casos en que la publicación es conjunta.

En la presente investigación se espera que en la medida que crece la intermediación de los países en la estructura de la red de colaboración regional crezca su impacto. Así, se define como segunda hipótesis que existe una correlación positiva entre la intermediación del país latinoamericano en la estructura de la red de colaboración regional y el impacto de sus artículos sobre biotecnología.

\section{Relación de la proximidad con el impacto}

Abbasi, Altmann y Hossain (2011) reportan la existencia de una correlación positiva entre la proximidad de los autores en la estructura de la red de colaboración y su impacto (índice $g$ ) $r=, 055, p<0,05$. Badar, Hite y Badir (2014) reportaron que la no existencia de influencia de la intermediación de 2027 investigadores en la red de coautoría en la investigación en química en Pakis- 
tán no influye en su desempeño $(\beta=0,046, p>0,01)$. Para la presente investigación se define como tercera hipótesis que existe una correlación positiva entre la proximidad del país latinoamericano en la estructura de la red de colaboración regional y el impacto de sus artículos sobre biotecnología.

\section{Variable dependiente: el impacto de los artículos latinoamericanos sobre biotecnología}

El empleo de la producción científica y el número de citas que reciben los artículos como indicadores para medir el desempeño científico de investigadores, centros de investigación y universidades, así como su utilización como garante de fiel cumplimiento para asignar recursos y lograr procesos de acreditación institucional, ha traído consigo un creciente interés en su estudio.

Actualmente los académicos entienden que su carrera, salario y promociones dependen del impacto que los resultados de sus investigaciones ejercen en sus pares académicos (Cordero-Villafáfila y Ramos-Brieva, 2014; Finkel, 2014). En la ciencia en general, para medir la influencia de un autor en el resto de la comunidad se tiene en cuenta la frecuencia en que sus trabajos son citados por sus pares académicos. Así, se le confiere una gran importancia al número de citas que un autor recibe para medir su desempeño y para asignar categorías docentes. Por ejemplo, en la gerencia se ha demostrado que existe una correlación positiva entre el número de citas de un investigador y el salario que percibe (Gómez-Mejía y Balkin, 1992).

La relación entre la colaboración y el impacto de las publicaciones ha sido analizada por varios autores (Gazni y Thelwall, 2014; Glänzel, 2002; Li, Liao y Yen, 2013; Yu et al., 2014). Desde que se creó el Science Citation Index existe consenso en la comunidad científica internacional sobre la importancia de medir el impacto de las publicaciones, lo cual se ha realizado, principalmente, mediante el recuento de las citas de los artículos publicados por un autor. La investigadora Renata Tagliacozzo (1977) en su artículo "Self-Citations in Scientific Literature" alertó sobre el sesgo que introducen las autocitas en el impacto. Así, en la última década ha crecido el número de críticas a la medición del desempeño a través del recuento de citas. El aspecto más mencionado es el efecto de inflación que ocasionan las autocitas en el impacto (Chang, McAleer y Oxley, 2013; Diekhoff, Schlattmann y Dewey, 2013; Ferrara y Romero, 2013). Esta situación ha traído consigo no sólo el cuestionamiento de la confiabilidad de la utilización del recuento de citas como indicador de desempeño sino de la determinación de la influencia real de un investigador sobre el resto de la comunidad científica de su área cuando el número de autocitas de sus artículos es elevado. 
Para mejorar la confiabilidad de la medición del impacto de los artículos como indicador de desempeño se han generado diferentes índices. El más popular es el creado por Jorge Hirsch (2005), el cual fue complementado posteriormente, entre otros, con el índice $e$ (Zhang, 2009), el índice $b$ descontado (Ferrara y Romero, 2013) y el índice $g$ (Egghe, 2006). Este último se ha convertido en uno de los más aceptados por la comunidad académica ya que supera la limitación del índice $b$ al no premiar a los artículos con un elevado número de citas. No obstante, presenta una situación que puede afectar la confiabilidad en la medición del impacto a nivel de países porque tiende a favorecer a los países con mayor producción científica. Así, países latinoamericanos grandes como Brasil se benefician por su ventaja de poseer una producción científica muy superior al resto de los países de la región.

Para superar las limitaciones mencionadas, en la presente investigación se ha seleccionado el índice Crown (Waltman et al., 2011a, 2011b) para medir el impacto de los países latinoamericanos en la investigación sobre biotecnología, debido a que supera la limitación de los índices de Hirsch con sus variaciones y el índice $g$ de favorecer a los países con mayor producción científica.

Para la generación del índice Crown se tienen en cuenta no sólo las citas recibidas por un país, sino también la relevancia de las revistas que las emiten. La composición del conjunto de publicaciones se pondera en relación con la media, para el presente estudio con el área de biotecnología. Posteriormente se normaliza el impacto de manera que países con impacto normalizado en la media mundial tendrán valor 1 . Los trabajos de dicho país se han publicado en revistas que se encuentran en la media de impacto de su categoría. Así, el impacto normalizado superior a 1 indica medias de impacto superiores a la categoría de la revista, impactos normalizados inferiores a 1 indican medias de impacto inferiores a la categoría de la revista. El procedimiento para calcular el índice Crown se explica en Waltman et al. (2011a, 2011b) y Moed (2010).

\section{Variables independientes}

El grado de centralidad de los paises latinoamericanos en la red de colaboración

El análisis de redes sociales ha despertado considerable interés en los últimos años y juega un papel importante en muchas disciplinas (Liu et al., 2005). El análisis de redes sociales es una estrategia poderosa para las ciencias de la información (Abbasi, Hossain y Leydesdorff, 2012). Otte y Rousseau (2002) definen el análisis de redes sociales como una estrategia amplia para investigar las estructuras sociales. El incremento de la complejidad de 
los problemas y la dinámica de crecimiento sostenido del conocimiento ha provocado un creciente interés sobre la estructura y la sociología de la colaboración científica (Racherla y Hu, 2010).

El principio básico del análisis de redes sociales es cuantificar las relaciones que se establecen entre los participantes en un grupo. Dichas relaciones conforman una estructura. En el análisis de las publicaciones científicas las redes se pueden analizar mediante el análisis de las coautorías de los artículos. Así, cuando autores procedentes de dos países unen esfuerzos en una publicación crean un lazo entre ambos países. Entre mayores relaciones recibe un país mayor será su centralidad en la estructura de la red.

La medida de centralidad es un conjunto de algoritmos que se calculan en la red y que permiten determinar tanto su estructura como la posición de cada vértice (entiéndase cada país) en dicha estructura (De Nooy, Mrvar y Batagelj, 2008). La medida de la centralidad proviene de los trabajos de Bavelas $(1948 ; 1950)$ y en la actualidad existen varias medidas para analizar la influencia de un actor en la estructura de una red. Las más empleadas son el grado, la intermediación (Freeman, 1977), la proximidad y la información (Stephenson y Zelen, 1989). Para el presente estudio se utiliza el grado de centralidad y la intermediación de cada país latinoamericano en la red de colaboración en la investigación sobre biotecnología.

El grado de centralidad recoge la medida de la centralidad total de un país latinoamericano en la red de investigación sobre biotecnología a partir de los enlaces que el mismo establece con el resto de los países participantes a través de la coautoría de los artículos. El resultado permite establecer los países que ocupan las posiciones centrales en la estructura de la red determinando cuáles son los que se relacionan más con el resto en la estructura de la red. Para calcular el grado se utiliza la siguiente fórmula:

$$
\mathrm{C}_{D}^{\prime}\left(n_{i}\right)=\mathrm{cd}\left(n_{i}\right) / \mathrm{g}-1
$$

donde $\mathrm{C}_{D}{ }_{D}\left(n_{i}\right)$ es el grado de centralidad del país $n_{i}, \operatorname{cd}\left(n_{i}\right)$ es la cantidad de países participantes en los artículos sobre biotecnología publicados por un país latinoamericano en la base de datos ISI y g-1 es la cantidad total de países presentes en la red excluyendo el país analizado.

\section{La intermediación de los países latinoamericanos en la red de colaboración}

La intermediación es una medida de centralidad que se basa en la distancia más próxima entre los diferentes países que participan en la estructura de colaboración. Así, el país más central teniendo en cuenta la intermediación 
es aquél que está ubicado en el camino más próximo entre el resto de los países en la estructura de la red. Es decir, se convierte en el puente de comunicación entre muchos otros países. Para calcular la intermediación se emplea la siguiente fórmula:

$$
\mathrm{C}_{D}^{\prime}\left(n_{i}\right)=\frac{\mathrm{C}_{B}\left(n_{i}\right)}{[(g-1)(g-2) / 2)]}
$$

donde $\mathrm{C}_{B}\left(n_{i}\right)$ es la suma de las probabilidades de que un país $\left(n_{i}\right)$ aparezca como puente en la ruta más próxima entre otros países y $[(g-1)(g-2) / 2)]$ es el número total de caminos más próximos entre el resto de los países en la red no incluyendo el país $\left(n_{i}\right)$.

\section{La proximidad de los paises latinoamericanos en la red de colaboración}

La medida de centralidad proximidad fue propuesta por Freeman (1979) para medir la centralidad entre varios nodos en una red. Así, un nodo es central si es el más próximo al resto de los nodos en la red. Para calcular la proximidad de los países en la presente investigación se ha empleado la fórmula de Abbasi, Altmann y Hossain (2011).

Para calcular las tres medidas de centralidad de los países latinoamericanos en la red de colaboración en la investigación sobre biotecnología, una vez construida la matriz de modo uno se empleó el programa informático Pajek (Batagelj y Mvar, 1998).

\section{DATOS Y FUENTES DE INFORMACIÓN}

En la presente investigación se han empleado técnicas del análisis de redes sociales para develar y representar gráficamente la estructura latente de la red latinoamericana de colaboración internacional en la investigación sobre biotecnología. El procedimiento empleado consta de tres pasos:

1. Se realizó una búsqueda en la base de datos ISI Web of Science según esta estrategia: búsqueda avanzada CU = (país) y categoría de la Web of Science (WC) = Biotechnology E Applied Microbiology. Marco temporal: desde el primero de enero de 1988 hasta el 31 de diciembre de 2012. Idioma: todos. Bases de datos de citaciones: Science Citation Index Expanded. Tipo de documento: artículo. Para cuantificar las re- 
laciones entre los países latinoamericanos en las publicaciones sobre biotecnología se jerarquizaron los resultados por países y territorios con un valor mínimo de 1 .

2. Se creó una matriz de modo uno $(n \times n)$ colocando como unidad de análisis (filas) los 21 países latinoamericanos que publicaron al menos un artículo sobre biotecnología en el marco temporal analizado, y como variables (columnas) los países latinoamericanos con los cuales estos países colaboraron en sus publicaciones. Durante la codificación de los datos se encontraron dos situaciones: 1) el autor firma en representación de un país (en estos casos se asignó un punto a cada país representado por cada autor firmante) y 2) se encontraron artículos $(4.07 \%)$ en los que el autor firma representando a más de un país. En la codificación de estos registros se ajustó el número de apariciones con el procedimiento descrito por Heck y Cooley (1988), Morrison e Inkpen (1991) y Shane (1997). El referido procedimiento establece que para un artículo que un autor representa a dos países, cada país recibe 0,5 puntos, si firma por tres, $1 / 3$ y así sucesivamente.

3. Tercero: se representó gráficamente la estructura de la red latinoamericana de colaboración en la investigación sobre biotecnología mediante el software Pajek y se utilizó la técnica Kamada-Kawai (1989) para la distribución espacial de los países en el gráfico. Una vez determinada la estructura latente de la red de colaboración se calculó el grado de centralidad de cada país en la estructura de la red mediante la utilización del comando partition del software Pajek.

\section{ANÁLISIS Y DISCUSIÓN}

En la Tabla 2 se muestran los datos sobre los indicadores bibliométricos de las variables del estudio. Como se puede apreciar cuatro países acumulan el 87.29 \% de la producción científica sobre biotecnología entre 1988 y 2012. Los países son Argentina, Brasil, Chile y México. Los artículos sobre biotecnología de estos países son los que mayor impacto poseen en la etapa analizada. Este resultado muestra la ventaja de los países con mayor desarrollo económico y científico, pues son los que más artículos producen.

Sin embargo, cuando se normaliza el impacto mediante el indicador Crown sólo dos países pequeños poseen impactos superiores a la media mundial, ellos son Bolivia y Costa Rica. Este resultado muestra la ventaja del indicador Crown para comparar el impacto de los países sin que el efecto tamaño introduzca sesgos en los resultados. 
Tabla 2. Descriptivos sobre la investigación de países latinoamericanos sobre biotecnología.

\begin{tabular}{|c|c|c|c|c|c|c|}
\hline País & $\begin{array}{c}\text { Producción } \\
\text { científica }\end{array}$ & $\begin{array}{l}\text { Número } \\
\text { de citas }\end{array}$ & Grado & Intermediación & Proximidad & Índice Crown \\
\hline Argentina & 2262 & 30322 & 0,60 & 0,087 & 0,536 & 0,75 \\
\hline Bolivia & 48 & 715 & 0,30 & 0,015 & 0,409 & 1,05 \\
\hline Brasil & 6203 & 82508 & 0,65 & 0,082 & 0,576 & 0,54 \\
\hline Colombia & 332 & 4779 & 0,50 & 0,016 & 0,501 & 0,5 \\
\hline Chile & 994 & 14816 & 0,55 & 0,035 & 0,518 & 0,84 \\
\hline Costa Rica & 84 & 1799 & 0,55 & 0,062 & 0,501 & 1,71 \\
\hline Cuba & 659 & 8490 & 0,60 & 0,082 & 0,501 & 0,28 \\
\hline Ecuador & 24 & 533 & 0,20 & 0 & 0,345 & 0,49 \\
\hline Guadalupe & 28 & 495 & 0,10 & 0,027 & 0,324 & 0,59 \\
\hline Guatemala & 20 & 204 & 0,15 & 0 & 0,361 & 0 \\
\hline México & 2913 & 41949 & 0,55 & 0,097 & 0,518 & 0,73 \\
\hline Nicaragua & 12 & 376 & 0,10 & 0 & 0,324 & 0,42 \\
\hline Panamá & 23 & 694 & 0,25 & 0,01 & 0,388 & 0,41 \\
\hline Paraguay & 5 & 85 & 0,05 & 0 & 0,33 & 0,06 \\
\hline Perú & 102 & 1578 & 0,40 & 0,001 & 0,444 & 0,78 \\
\hline Trin Tobago & 45 & 744 & 0,15 & 0,001 & 0,311 & 0,49 \\
\hline Uruguay & 192 & 3552 & 0,05 & 0,001 & 0,33 & 0,68 \\
\hline Venezuela & 198 & 2875 & 0,35 & 0,022 & 0,444 & 0,62 \\
\hline Haití & 1 & 4 & 0,05 & 0 & 0 & 0,81 \\
\hline Honduras & 11 & 137 & 0,05 & 0 & 0,317 & 0,16 \\
\hline Barbados & 9 & 55 & 0,05 & 0 & 0 & 0,47 \\
\hline
\end{tabular}

En la Figura 1 se muestra la estructura de la red latinoamericana de colaboración científica en la investigación sobre biotecnología. Los países con mayor grado de centralidad son Brasil, Cuba, Argentina, Chile, Costa Rica y México, los que poseen menos grado de centralidad en la red son Barbados, Haití y Honduras, que dependen de la colaboración para mantenerse conectados al núcleo de la red. 


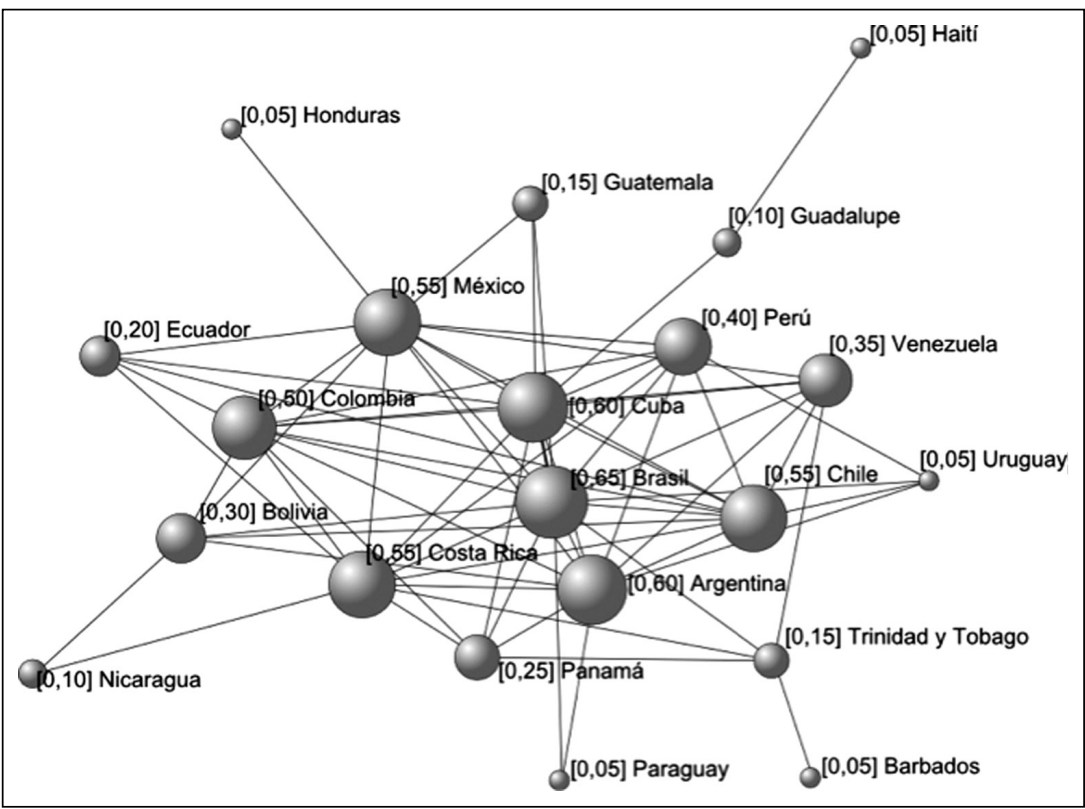

Figura 1. Red de colaboración latinoamericana en la investigación sobre biotecnología Nota: valores del grado de centralidad aparece entre corchetes

La variable dependiente impacto tiene un valor mínimo de 0 y máximo de 1,710, y una media de ,589. El resultado de la prueba Shapiro Wilki (W-Statistic $=0,915, p=0,029)$ muestra que esta variable posee una distribución no normal. En la Tabla 3 se pueden observar los valores de la media y de la desviación típica.

Como las cuatro variables del estudio no cumplen el supuesto de normalidad de la distribución se realizó una prueba Spearman Rho para determinar si la variable impacto está relacionada con las medidas de centralidad grado, intermediación y proximidad. La Tabla 3 muestra los resultados. Como se puede apreciar las variables impacto y grado de centralidad no están relacionadas $(0,369, p<0,099)$ por lo que no se valida la primera hipótesis. El resultado es opuesto a los reportados por Abbasi, Chung y Hossain (2012) y Abbasi, Altmann y Hossain (2011) midiendo el impacto a través del índice $g$, y de Cimenler, Reeves y Skvoretz (2014) midiendo el impacto mediante el índice $h$, y de Badar, Hite y Badir $(2012,2014)$ con la producción científica. Los resultados obtenidos pueden tomarse como evidencia de la influencia del efecto tamaño de los países sobre el impacto si éste se mide a través de los índices Hirsch, $g$ o Hirsch descontado. Asimismo, el resultado demuestra el efecto neutralizador de indicador Crown en la desventaja de los índices previos de favorecer a las instituciones o países más grandes. 
Tabla 3. Intercorrelaciones, medias y desviación típica de impacto y las medidas de centralidad $(N=14,173)$.

\begin{tabular}{|l|c|c|c|c|c|}
\hline & Grado & Intermediación & Proximidad & Media & Desviación típica \\
\hline Impacto &, 369 &, $473^{\star}$ &, 364 & 0,590 & 0,366 \\
\hline Grado & &, $847^{\star *}$ &, $934^{\star *}$ & 0,298 & 0,223 \\
\hline Intermediación & & &, $819^{\star \star}$ & 0,026 & 0,034 \\
\hline Proximidad & & & & 0,380 & 0,152 \\
\hline
\end{tabular}

${ }^{*} p<0,05,{ }^{* *} p<0,001$.

Se encontró una correlación positiva del impacto con la intermediación $(0,473, p<0,05)$ de los países en la estructura de colaboración regional. Este valor se interpreta como un efecto mediano de acuerdo con los valores base de Cohen (1988). El resultado valida la segunda hipótesis. El hallazgo es opuesto al reportado por Badar, Hite y Badir (2014) para el área de química en Pakistán, quienes encontraron la no existencia de correlación entre intermediación e impacto medido como la producción científica normalizada. Así en la presente investigación se corroboran los hallazgos de Abbasi, Chung y Hossain (2012) para el índice $g$ aunque el valor de la correlación es inferior a los reportados por los citados autores. Al igual que para la primera hipótesis la relación puede ser menor debido a la neutralización del efecto tamaño de los países a través del indicador Crown. El presente hallazgo también corrobora los resultados encontrados por Cimenler, Reeves y Skvoretz (2014).

No se encontró la existencia de relación entre el impacto y la proximidad de los países $(0,364, p<0,105)$, por lo que la tercera hipótesis no se valida. El resultado encontrado es similar al reportado por Badar, Hite y Badir (2014) y opuesto al presentado por Abbasi, Altmann y Hossain (2011).

\section{Conclusiones}

Los resultados de la presente investigación muestran el incremento de la atención que han brindado los países latinoamericanos a la investigación sobre biotecnología en los últimos años. Este aspecto queda evidenciado en el crecimiento en 12 veces la producción científica sobre esta área de investigación del 2010 en relación con la de 1990. Brasil, Argentina, México y Chile son los países con mayor producción científica en esta área de investigación.

En este incremento significativo ha jugado un papel esencial la colaboración académica con países más desarrollados en la investigación sobre biotecnología, principalmente con los ubicados en el centro de la red de investigación de esta disciplina, tales como Estados Unidos, Japón, Alemania, 
Inglaterra, España y Francia. Estos elementos evidencian la necesidad de encaminar la política científica de la región a fomentar los vínculos con los principales centros de investigación de estos países como una estrategia favorecedora para incrementar el impacto de los resultados del área en la investigación sobre biotecnología.

Cuando se analizan los valores de centralidad de cada país latinoamericano en la estructura de la red de colaboración regional, Cuba y México aparecen segundos después de Brasil. El resultado muestra que existe una relación positiva entre el impacto y la intermediación de los países en la estructura de la red de colaboración regional, no así con las medidas de centralidad grado y proximidad. Este resultado demuestra que en una red de colaboración científica no sólo es importante tener una buena producción científica e impacto sino la capacidad de convertirse en mediador o enlace en el establecimiento de la colaboración entre los países participantes en la estructura de la red. Así, los investigadores de los países que sirven de puente en la comunicación aumentan su capacidad de absorción de recursos, nuevas tecnologías, acceso a recursos escasos y laboratorios con tecnología actualizada.

Los elementos expuestos contribuyen con el aumento de la reputación de los investigadores de los países participantes en la red ante el resto de la comunidad académica. Esto favorece el incremento de los lazos de colaboración con investigadores e instituciones de mayor importancia mundial, y facilita la participación de estos países en proyectos de mayor relevancia para la solución de los problemas más complejos en el área de biotecnología.

Finalmente, se ha demostrado que el indicador Crown es una medida eficaz en la comparación del impacto de los países reduciendo la desventaja que poseen los más pequeños en relación con los países más desarrollados. Esta conclusión se sustenta en que en el presente estudio dos países latinoamericanos pequeños son los que poseen un impacto superior a la media mundial en el impacto en la investigación sobre biotecnología.

La presente investigación puede ser complementada con el análisis de la red de colaboración latinoamericana con los países fuera de la región y el estudio de la red de colaboración a nivel mundial para contrastar los resultados encontrados.

\section{Agradecimientos}

Los autores agradecen a tres evaluadores anónimos por sus sugerencias y guía, las cuales han contribuido con la mejora en la presentación de los resultados de la investigación. Los autores agradecen al Profesor Félix de Moya Anegón del Grupo Scimago por facilitarnos los datos del índice 
Crown para los análisis, así como por sus intuitivos comentarios sobre el manuscrito.

\section{REFERENCIAS}

Abbasi, A., J. Altmann y L. Hossain. 2011. "Identifying the effects of co-authorship networks on the performance of scholars: A correlation and regression analysis of performance measures and social network analysis measures". Journal of Informetrics 5 (4): 594 607. doi: 10.1016/j.joi.2011.05.007

Abbasi, A., K. S. Chung y L. Hossain. 2012. "Egocentric analysis of co-authorship network structure, position and performance". Information Processing \& Management 48 (4): 671-679. doi: 10.1016/j.ipm.2011.09.001

Abbasi, A., L. Hossain y L. Leydesdorff. 2012. "Betweenness centrality as a driver of preferential attachment in the evolution of research collaboration networks". Journal of Informetrics 6 (3): 403 412. doi: 10.1016/j.joi.2012.01.002

Badar, K., J. M. Hite y Y. F. Badir. 2012. “Examining the relationship of co-authorship network centrality and gender on academic research performance: the case of chemistry researchers in Pakistan." Scientometrics 94 (2): 755-775. doi: 10.1007/s11192-012-07 64-z

J. M. Hite y Y. F. Badir. 2014. "The moderating roles of academic age and institutional sector on the relationship between co-authorship network centrality and academic research performance." Aslib Journal of Information Management 66 (1): 38-53. doi: 10.1108/ajim-05-2013-0040

Ball, P. 2005. "Index aims for fair ranking of scientists." Nature 436 (7053): 900. doi: 10.1038/436900a

Batagelj, V. y A. Mrvar. 1996. Pajek (Version 1.24) [Free for noncomercial use]: Vladimir Batagelj and Andrej Mrvar.

— y A. Mrvar. 1998. "Pajek. Program for large network analysis." Connections 21: 47-57.

Bavelas, A. 1948. "A Mathematical model for group structure." Human Organizations 7: 6-30.

1950. “Communication patterns in task-oriented groups.” Journal of the Acoustical Society of America 22: 271-282.

Cimenler, O., K. A. Reeves y J. Skvoretz. 2014. "A regression analysis of researchers' social network metrics on their citation performance in a college of engineering." Journal of Informetrics 8 (3): 667-682. doi: 10.1016/j.joi.2014.06.004

Cohen, J. 1988. Statistical Power and Analysis for the Behavioral Sciences. 2a. ed. New Jersey: Lawrence Erlbaum. 
Cordero-Villafáfila, A. y J. A. Ramos-Brieva. 2014. "La evaluación del factor de impacto individual de investigadores y centros de investigación utilizando el algoritmo RC." Revista de Psiquiatría y Salud Mental. doi: 10.1016/j.rpsm.2013.11.002

Costa, B. M. G., E. da Silva Pedro y G. R. Macedo. 2012. “Scientific collaboration in biotechnology: the case of the northeast region in Brazil." Scientometrics 95 (2): 571-592. doi: 10.1007/s11192-0120924-1

Chang, C. L., M. McAleer y L. Oxley. 2013. "Coercive journal self citations, impact factor, Journal Influence and Article Influence." Mathematics and Computers in Simulation 93: 190-197. doi: 10.1016/j.matcom.2013.04.006

Dalpé, R. 2002. "Bibliometric analysis of biotechnology." Scientometrics 55 (2): 189-213. doi: 10.1023/a:1019663607103

De Nooy, W., A. Mrvar y V. Batagelj. 2008. Exploratory Social Network Analysis with Pajek. New York: Cambridge University Press.

Diekhoff, T., P. Schlattmann y M. Dewey. 2013. "Impact of Article Language in Multi-Language Medical Journals - a Bibliometric Analysis of Self-Citations and Impact Factor." Plos One 8 (10): 8. doi: 10.1371/journal.pone.0076816

Egghe, L. 2006. "Theory and practise of the g-index." Scientometrics 69 (1): 131-152. doi: 10.1007/s11192-006-0144-7

Eslami, H., A. Ebadi y A. Schiffauerova. 2013. "Effect of collaboration network structure on knowledge creation and technological performance: the case of biotechnology in Canada." Scientometrics 97 (1): 99-119. doi: 10.1007/s11192-013-1069-6

Ferrara, E. y A. E. Romero. 2013. "Scientific Impact Evaluation and the Effect of Self-Citations: Mitigating the Bias by Discounting the h-Index." Journal of the American Society for Information Science and Technology 64 (11): 2332-2339. doi: 10.1002/asi.22976

Finkel, A. 2014. "Perspective: powering up citations." Nature 511 (7510): S77. doi: 10.1038/511S77a

Freeman, L. 1977. "A set of measures of centrality based on betwenness." Sociometry 40 (1): 5-41.

Freeman, L. C. 1979. "Centrality in social networks conceptual clarification." Social Networks 1 (3): 215-239.

Frenken, K., W. Hoolzl y F. de Vor. 2005. “The citation impact of research collaborations: the case of European biotechnology and applied microbiology (1988-2002)." Journal of Engineering and Technology Management 22: 9-30.

Gazni, A. y M. Thelwall. 2014. "The long-term influence of collaboration on citation patterns." Research Evaluation. doi: 10.1093/rese $\mathrm{val} / \mathrm{rvu} 014$

Glänzel, W. 2002. "Coauthorship Patterns and Trends in the Sciences (1980-1998) :A Bibliometric Study with Implications for Database Indexing and Search Strategies." Library Trends 50 (3): 461-473. 
Glänzel, W. y B. Thijs. 2004. "Does co-authorship inflate the share of self-citations?” Scientometrics 61 (3): 395-404.

y P. Zhou. 2010. "Publication activity, citation impact and bi-directional links between publications and patents in biotechnology." Scientometrics 86 (2): 505-525. doi: 10.1007/s11192-010-0269-6

Gómez-Mejía, L. R. y D. B. Balkin. 1992. "Determinants of faculty pay: An agency theory perspective." Academy of Management Journal 35 (5): 921-955.

Hartley, J. 2012. "To cite or not to cite: author self-citations and the impact factor." Scientometrics 92 (2): 313-317. doi: 10.1007/s11192011-0568-6

Heck, J. y P. Cooley. 1988. "Most frequent contributors to the finance literature." Financial Management, Autumn: 100-108.

Hirsch, J. E. 2005. "An index to quantify an individual's scientific research output." Proc Natl Acad Sci US A 102 (46): 16569-16572. doi: 10.1073/pnas.0507655102

Huang, M.-H., S.-H. Chen, C.-Y. Lin y D.-Z. Chen. 2013. "Exploring temporal relationships between scientific and technical fronts: a case of biotechnology field." Scientometrics 98 (2): 1085-1100. doi: 10.1007/s11192-013-1054-0

Kamada, T. y S. Kawai. 1989. "An algorithm for drawing general undirected graphs.” Processing Letters 31 (1): 7-15.

Li, E. Y., C. H. Liao y H. R. Yen. 2013. "Co-authorship networks and research impact: A social capital perspective.” Research Policy 42 (9): 1515-1530. doi: 10.1016/j.respol.2013.06.012

Liu, X., J. Bollen, M. L. Nelsonb y H. Van de Sompel. 2005. "Co-authorship networ ks in the digital library research community." Information Processing \& Management 41: 1462-1480.

McCain, K. W. 1995. “The structure of biotechnology R \& D." Scientometrics 32 (2): 153-175. doi: 10.1007/bf02016892

Moed, H. F. 2010. "CWTS crown indicator measures citation impact of a research group's publication oeuvre." Journal of Informetrics 4 (3): 436-438. doi: 10.1016/j.joi.2010.03.009

Morrison, A. J. y A. C. Inkpen. 1991. "An Analysis of Significant Contributions to the International Business Literature.” Journal of International Business Studies 22 (1): 143-153.

Otte, E. y R. Rousseau. 2002. "Social network analysis: a powerful strategy, also for the information sciences." Journal of Information Science 28 (6): 441-453.

Racherla, P. y C. Hu. 2010. "A social network perspective of tourism research collaborations." Annals of Tourism Research 37 (4): 10121034. doi: 10.1016/j.annals.2010.03.008

Shane, S. A. 1997. "Who is Publishing the Entrepreneurship Research?” Journal of Management 23: 83-95.

Stephenson, K. y M. Zelen. 1989. "Rethinking centrality: Method and applications.” Social Networks 11 (1): 1-37. 
Tagliacozzo, R. 1977. "Self-Citations in Scientific Literature.” Journal of Documentation 33 (4): 251-265. doi: 10.1108/eb026644

Torabian, R., A. Heidari, M. Shahrifar, E. Khodadi y S. A. E. Vardanjani. 2012. "The Relation between Self-Citation and Impact Factor in Medical Science Open Access Journals in ISI \& DOAJ Databases." Life Science Journal-Acta Zhengzhou University Overseas Edition 9 (4): 2206-2209.

Van Raan, A. F. J. 1998. "The influence of international collaboration on the impact of research results - Some simple mathematical considerations concerning the role of self-citations." Scientometrics 42 (3): 423-428.

Waltman, L., N. J. van Eck, T. N. van Leeuwen, M. S. Visser y A. F. J. van Raan. 2011a. "Towards a new crown indicator: an empirical analysis." Scientometrics 87 (3): 467-481. doi: 10.1007/s11192-0110354-5

- N. J. van Eck, T. N. van Leeuwen, M. S. Visser y A. F. J. van Raan. 2011b. "Towards a new crown indicator: Some theoretical considerations." Journal of Informetrics 5 (1): 37-47. doi: 10.1016/j. joi.2010.08.001

Yu, Q., H. Shao, C. Long y Z. Duan. 2014. "The Relationship Between Research Performance and International Research Collaboration in the C\&c Field." Experimental \& Clinical Cardiology 20 (6): $145-153$.

Zhang, C. T. 2009. "The e-index, complementing the h-index for excess citations.” Plos One 4 (5): e5429. doi: 10.1371/journal.pone. 0005429

Para citar este artículo:

Ronda Pupo, Guillermo Armando et al. 2016. "Correlación entre las medidas de centralidad de los países y el impacto de sus artículos. Caso de estudio de la investigación sobre biotecnología en Latino América." Investigación Bibliotecológica: Archivonomía, Bibliotecología e Información 69: 75-94. http://dx.doi.org/10.1016/j.ibbai. 2016.04.013 